\title{
A new approach for computing diode sputter-ion pump characteristics
}

\author{
H. Hartwig and J. S. Kouptsidis \\ Deutsches Elektronen-Synchroton DESY, Hamburg, Germany
}

(Received 22 May 1974; in final form 28 August 1974)

By using theoretical considerations combined with experimental results, a general approach for computing sputter-ion pump characteristics is given. This approach can be successfully applied to optimal design of distributed sputter-ion pumps in high energy accelerators. The deviation between computed and measured pumping speed values is less than $30 \%$.

\section{INTRODUCTION}

Despite the widespread use of sputter-ion pumps in almost all vacuum applications, our knowledge about the influence of the different pump parameters on the pumping speed is more empirical than fundamental. This is probably due to the complicated pumping mechanism of the sputter-ion pumps, which depends on the different discharge modes of the Penning cells, on the sputtering rate of the energetical ions impacting the cathode, and on physicochemical phenomena between gas molecules and the surfaces of both electrodes.

Besides the cathode material, the main significant parameters affecting the pumping speed of a sputter-ion pump are the magnetic field, the applied voltage, and the diameter of the cells. Fortunately for the designers of conventional sputter-ion pumps, the values of the magnetic field and the voltage are cost limited and can not be optimized. Almost all pumps work with magnetic fields of $1-2 \mathrm{kG}$ and voltages of $5-8 \mathrm{kV}$. The remaining parameter, the cell diameter, can be optimized experimentally.

Recently, sputter-ion pumps have found another application in particle accelerators, particularly in storage rings ${ }^{1-3}$ as distributed pumping units. The bending magnets of the particle accelerators provide the magnetic field required for the pump. The magnetic field of the bending magnets of a storage ring is proportional to the energy of the stored particles and generally ranges between 0.5 and $12 \mathrm{kG} .{ }^{4}$ In the case of electron storage rings, the gas desorption of the vacuum system is due mainly to the synchrotron radiation which depends on the energy and current of the stored electrons. ${ }^{5}$ Thus the distributed sputter-ion pump for a storage ring must be designed to perform optimally when the maximum gas desorption takes place in the accelerator. It must also provide a sufficiently large pumping speed over the whole operating range of the accelerator.

Unfortunately, the only existing empirical formalism for optimal sputter-ion pump design of Malev et al., ${ }^{1,21}$ shows discrepancies between measured and expected pumping speed when the magnetic field varies. Figure 1 shows these discrepancies as observed for prototypes of distributed pumps of the SPEAR ${ }^{24}$ storage ring. ${ }^{2}$ For these large cells $(\phi 2.5 \mathrm{~cm})$, working in high magnetic fields $(>10 \mathrm{kG})$ the deviation between calculated and measured pumping speed goes up to a factor of 5. In contrast to Malev's formalism, it is well-known that the pumping speed of a sputter-ion pump reaches a limit value when the magnetic field is increased. This limit is not due to the conductance of the pump elements, but it is an inherent property of the Penning discharge. ${ }^{6}$ Malev's formalism is an empirical result from pumping speed measurements made over a broad range of values of cell parameters such as diameter, applied voltage, and magnetic field. While making these large variations, the discharge takes place in different modes, and the measured pumping speed values can not be fitted by an empirical formalism

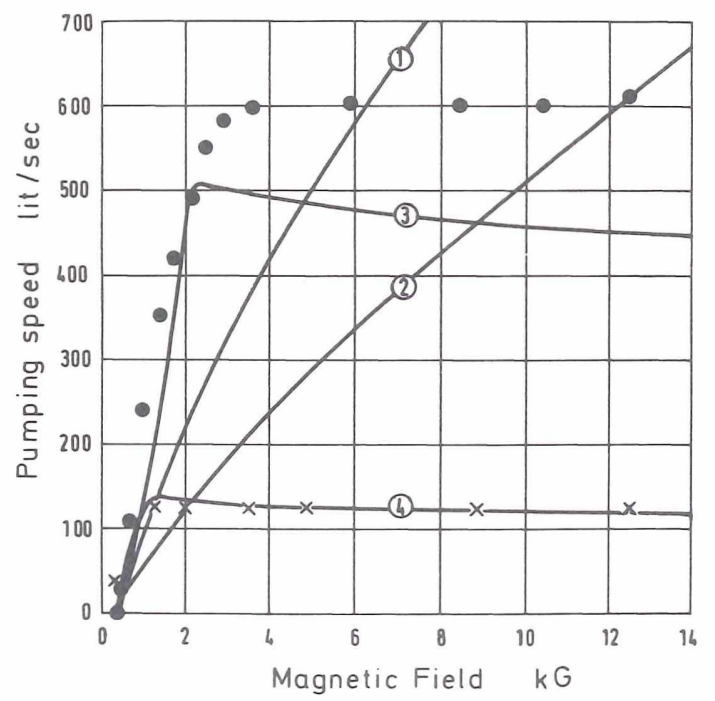

FIG. 1. Pumping speeds of SPEAR storage ring pumps. - - measured values with $1.25 \mathrm{~cm}$ diam. cells; $X$-measured values with $2.50 \mathrm{~cm}$ diam. dells; (1) - calc. pumping speed with Malev's formalism for $1.25 \mathrm{~cm}$ diam. cells; (2)-calc. pumping speed with Malev's formalism for $2.50 \mathrm{~cm}$ diam. cells; (3-calc. pumping speed as proposed in this paper for $1.25 \mathrm{~cm}$ diam. cells; (4)-calc. pumping speed as proposed in this paper for $2.50 \mathrm{~cm}$ diam. cells. 
without a classification in these modes. The different discharge modes and errors in the pumping speed measurements probably explain the observed discrepancies.

In this paper, a new semiempirical formalism to determine the characteristics of diode sputter-ion pumps is proposed. This formalism uses existing theories of the different discharge modes of a Penning cell ${ }^{6}$ combined with experimental results on the pumping speed of sputter-ion pumps obtained at DESY25 and other laboratories. ${ }^{2,6}$

\section{PUMPING SPEED AND DISCHARGE INTENSITY}

In the early works on sputter-ion pumps, it was suggested and experimentally proved that the pumping speed is closely related to discharge intensity $I / P$ ( $I=$ discharge current, $P=$ pressure).${ }^{7-11}$ This relationship is linear $(S=c I / P)$ for a given gas, and was used to determine the pumping speed especially at low pressures since the discharge intensity can easily be measured. The proportionality factor $c$ depends not only on the gas ${ }^{11}$ but also on the gas quantity pumped after bake-out of the pump. To avoid complications due to such saturation effects, we first deal with pumping speeds of virgin pumps after appropriate bake-out (more than $5 \mathrm{~h}$ at $350^{\circ} \mathrm{C}$ ).

The published $c$-values for nitrogen ${ }^{7,9-11}$ were obtained from measurements of the pumping speed $S$ under equilibrium conditions, the pump current $I$, and the pressure $P$ in the pump $(c=S P / I)$. Rutherford ${ }^{9}$ found experimentally that the $c$-value depends on the working voltage of the pump. The nitrogen $c$-values for a typical diode sputter-ion pump were 0.05 Torr $1 / \sec A$ at $3 \mathrm{kV}$ and 0.08 Torr $1 / \sec A$ at $6 \mathrm{kV}$. Bächler ${ }^{7}$ didn't emphasize this voltage dependence and reported a mean $c$-value of 0.07 Torr $1 / \sec A$ from measurements on a large number of different diode sputter-ion pumps working between 4 and $7 \mathrm{kV}$. The lowest nitrogen $c$-value of 0.027 Torr $1 / \sec A$ was measured by Dallos ${ }^{11}$ on a $15 \mathrm{l} / \mathrm{sec}$ sputter-ion pump working at $7.2 \mathrm{kV}$ in a magnetic field of $1.4 \mathrm{kG}$.

The large deviation between the two extreme published values $(0.027$ and 0.08 ) can not be explained only by assuming a $c$-dependence on the working voltage ${ }^{9}$ or on the electrode configuration. ${ }^{22}$ The two values were measured on similar sputter-ion pumps of the diode type working at almost the same voltage. Probably, an additional error due to the inherent incertitude of absolute pressure measurements is responsible for the large deviation of the $c$-value reported by the two authors. ${ }^{9,10}$

In this work, another procedure has been used to determine the $c$-value. The pumping speed was measured according to a proposal from Fischer and Mommsen ${ }^{12}$ and the required discharge intensity was calculated using Schuurman's theory on the Penning discharge. ${ }^{6}$ This was necessary because Schuurman's theory constitutes the basis of the proposed semiempirical formalism. Furthermore, deviations of the c-value from pressure measurement errors were considerably reduced due to the weak pressure dependence of the discharge intensity. The pumping speed measurements were carried out at DESY on a large number of conventional diode sputter-ion pumps with titanium cathodes working at different voltages $(3-8 \mathrm{kV}$ ) and having a nominal pumping speed for nitrogen between 150 and $800 \mathrm{l} / \mathrm{sec}$. In addition, the pumping speed of distributed sputter-ion pump prototypes were measured over a large magnetic field range $(0.5-13 \mathrm{kG})$. The published pumping speed measurements of the SPEAR storage ring distributed pumps were also taken into account. The best fit for all tested pumps is given by a $c$-value of $0.075 \operatorname{Torr} 1 / \sec A$. This value is nearly the same as measured by Bächler ${ }^{7}$ and lies between the two experimental values- 0.05 and 0.08 Torr $1 / \sec A-$ reported by Rutherford. ${ }^{9}$ This value is also satisfactory for differential diode sputter-ion pumps with cathodes having a tantalum proportion of less than $25 \%$.

At pressures higher than $10^{-7}$ Torr, the pumping speed doesn't increase at the rate of the calculated discharge intensity and results in a lowering of the $c$-value. The decrease of the pumping speed and the $c$-value for pressures higher than $10^{-7}$ Torr is probably due to the electron and ion impact-desorption on the gas covered pump electrodes. We have assumed that the electrode surface coverage increases according to a Langmuir isotherm. The validity of this assumption was not checked separately. Only the pressure dependence of the $c$-value was successfully fitted to an equation of the Langmuir isotherm type

$$
c=0.075\left(1-\frac{1.5 \times 10^{6} P}{1+4 \times 10^{6} P}\right) .
$$

This empirical relation is valid for the pressure region between $10^{-10}$ and $10^{-5}$ Torr. The equation

$$
S=0.075\left(1-\frac{1.5 \times 10^{6} P}{1+4 \times 10^{6} P}\right) I / P
$$

where $S$ is the pumping speed in $1 / \mathrm{sec}$, and $I / P$, the discharge intensity in A/Torr, is the basis of the semiempirical formalism. The constants and the following calculations are valid only for nitrogen.

Existing theories on the different discharge modes of a Penning cell given in Schuurman's survey ${ }^{6}$ were used to calculate the discharge intensity. Only two operation modes are significant for sputter-ion pumps working in the region of high and ultrahigh vacuum : the LMFmode (low magnetic field) and the HMF-mode (high magnetic field). These modes differ in their potential profile in the cell. In the LMF-mode, the potential profile in the cell is strongly deformed by the space charge of an electron cloud spread out over the whole volume of the cell. The HMF-mode, existing at high magnetic fields, is characterized by a field-free plasma region around the axis of the cell and by a cloud of electrons in the form of a sheath adjacent to the anode. 
The two modes can be easily distinguished in a typical diagram (Fig. 2) which shows the dependence between the discharge intensity and the magnetic field at constant pressure. At magnetic fields lower than the value $A$, no ignition of the discharge takes place and the discharge intensity is zero. The ignition point $A$ depends on the radius $r_{\mathrm{a}}$ of the anode cell and is given by the empirical equation ${ }^{1,21}$

$$
B_{i}=300 / r_{a} \text {, }
$$

where $r_{\mathrm{a}}$ equals cell radius in $\mathrm{cm}$ and $B_{i}$, the magnetic field at the ignition point in $G$. This empirical equation is valid mainly for high working voltages $\left(U_{\mathrm{a}}>3000 \mathrm{~V}\right)$.

The branch $\mathrm{AO}$ (Fig. 2) shows the typical increase of the discharge intensity with the magnetic field for the LMF-mode. The transition to the HMF-mode occurs where the discharge intensity has its maximum value (point $\mathrm{O}$ ). At low pressures $\left(\mathrm{p}<10^{-7}\right.$ Torr), the discharge intensity of the HMF-mode remains almost constant when the magnetic field is increased (branch OC). For $p>10^{-7}$ Torr, the discharge intensity decreases (branch OD) with increasing magnetic field. This decrease is accelerated for higher pressure values.

The magnetic field $B_{\text {tr }}$ at the transition point $O$ between the LMF- and HMF-modes is given by the equation ${ }^{6}$

$$
B_{t_{\mathrm{r}}}{ }^{2}=\frac{30.3 U_{\mathrm{a}}}{r_{\mathrm{a}}{ }^{2}\left(\nu_{i} / \nu_{c}\right)},
$$

with $U_{\mathrm{a}}$ as applied voltage in $V, r_{\mathrm{a}}$, cell radius in $\mathrm{cm}$, and $B_{\mathrm{tr}}$ in $G . \nu_{i} / \nu_{c}$ is the ionization probability of an electron in a collision with a gas molecule $\left(\nu_{i}=\right.$ ionization frequency, $\nu_{c}=$ collision frequency).

The discharge intensity of one cell in the LMF-mode is given by the equation ${ }^{6}$

$$
I / P=7.7 \times 10^{-4}\left(\nu_{i} / \nu_{c}\right)^{2} l r_{\mathrm{a}}{ }^{2} B^{2} .
$$

$I / P$ is in $\mathrm{A} /$ Torr, $l$ is the effective height of the cell in $\mathrm{cm}$, and $B$ is the magnetic field in $\mathrm{G}$. The effective cell height is greater than the natural one due to extension of the discharge in the space between anode and cathode by axial oscillations of the avalanche electrons. An increase of the natural cell height by $25 \%$ of sum of the gaps between anode and cathodes seems to present the effective anode height. This equation is valid only above the ignition point, i.e., for magnetic fields greater than $B_{i}$ (Fig. 2). To avoid discontinuity of the discharge intensity function at this point, we take a linear rise starting at $B_{i}$ and merging tangentially into the parabolic curve (Eq. 4). Thus the LMF-mode between the magnetic fields $B_{i}$ and $2 B_{i}$ can be given by the linear equation

$$
I / P=3.1 \times 10^{-3}\left(\nu_{i} / \nu_{c}\right)^{2}{ }^{2} r_{\mathrm{a}}{ }^{2} B_{i}\left(B-B_{i}\right) .
$$

Equation (4) must be used to calculate the discharge intensity for magnetic fields greater than $2 B_{i}$.

In order to reduce the computational effort to a minimum we have assumed that in the whole region of the HMF-mode the discharge intensity takes the
FIG. 2. A typical diagram of the discharge intensity dependence on the magnetic field at constant pressure. The brance AO is characteristic for the LMF-mode. At low pressures $\left(p<10^{-7}\right.$ Torr), the HMF-mode (OC) shows an almost constant discharge intensity. For high pressures $\quad\left(p>10^{-7}\right.$ Torr), the HMF-mode is given by the curve OD.

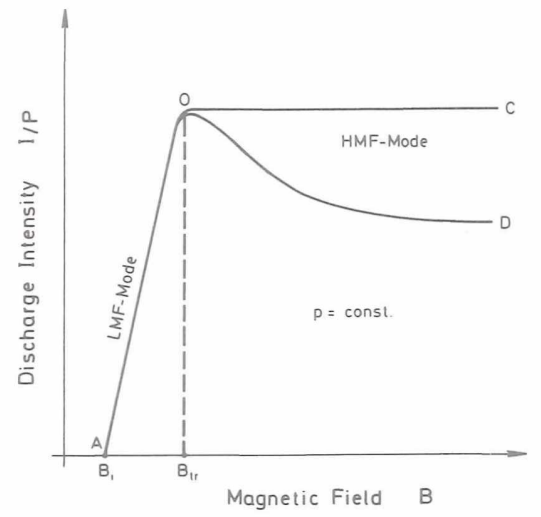

transition point value. This assumption has been confirmed experimentally ${ }^{6}$ for pressures below $10^{-7}$ Torr. The deviation from this assumption at pressures higher than $10^{-7}$ Torr is corrected by the empirical factor

$$
\left\{1-1.5 \times 10^{4}\left[\left(B-B_{\text {tr }}\right) r_{\mathrm{a}} P\right]^{\frac{1}{2}} / U_{\mathrm{a}}\right\} .
$$

Thus the following equation was assumed for the HMF-mode :

$$
\begin{aligned}
I / P=2.3 \times 10^{-2}\left(\nu_{i} / \nu_{c}\right) l U_{\mathrm{a}} \\
\\
\times\left\{1-1.5 \times 10^{4}\left[\left(B-B_{\text {tr }}\right) r_{\mathrm{a}} P\right]^{\frac{1}{2}} / U_{\mathrm{a}}\right\} .
\end{aligned}
$$

It is well known ${ }^{11}$ that the discharge current of a sputterion pump is not proportional to the pressure, but rather a function of the type $k p^{\alpha}$ where the exponent $\alpha$ is slightly greater than 1 . This typical dependence causes a decrease of the pumping speed at lower pressure. Thus the discharge intensity and the pumping speed show a pressure dependence of the type $k^{\prime} p^{\alpha-1}{ }^{11}$ The anomalous diffusion of the avalanche electrons across the magnetic field, owing to collective interactions in the discharge, is mainly responsible for this dependence.

In order to introduce this pressure dependence of the discharge intensity in Eqs. (3)-(6), classical electron diffusion in the discharge, we assumed a pressure dependence of the ionization probability $\left(\nu_{i} / \nu_{c}\right)$. This assumption does not have any deep physical meaning; rather, it is a consequence of the attempt to consider the component of the anomalous diffusion of the discharge electrons by using theories of the classical mobility. From measurements on typical diode sputterion pumps, working in the LMF-mode region, it has been found that the discharge current shows the following pressure dependence:

$$
I \propto P^{1.2} .
$$

Introducing this relation into the Eq. (4) results in a smooth pressure dependence of the ionization probability

$$
\nu_{i} / \nu_{c} \propto P^{0.1} .
$$

By relating this dependence to pumping speed measurements, it was possible to determine the proportionality factor in this equation

$$
\nu_{i} / \nu_{c}=0.52 \times P^{0.1} .
$$

Equation (9) is plotted in Fig. 3 together with measured 


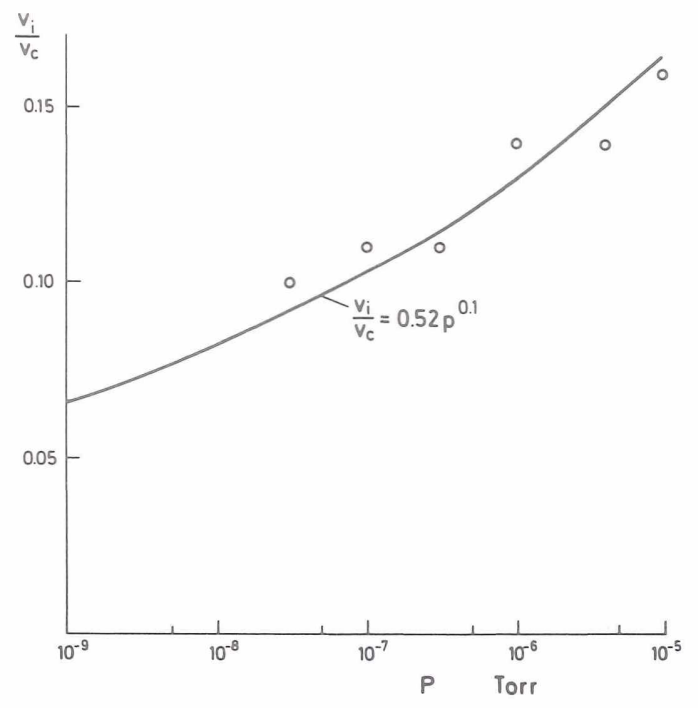

FIG. 3. Measured and proposed pressure dependence of the ionization probability $\nu_{i} / \nu_{c}{ }^{6}$

values of the ionization probability given by Schuurman. ${ }^{6}$ A good agreement between proposed and measured values of the ionization probability can be observed.

In practical cases, the magnetic field lines are not parallel to the axis of the cell due to misalignment of the cell or to inhomogeneities of the magnetic field. To calculate the influence of this misalignment, we assume that the electrons of the discharge build up a cylindrical cloud along the magnetic field lines through the center of the cell. A misalignment angle of $\phi$ limits the electron cloud to a radius $r_{\phi}$ smaller than $r_{\mathrm{a}}$ (Fig. 4). From geometry, the following relation results for $r_{\phi}$ :

$$
r_{\phi}=r_{\mathrm{a}} \cos \phi-0.5 l_{\mathrm{a}} \sin \phi,
$$

where $l_{\mathrm{a}}$ is the geometrical height of the cell. Using the effective radius of the cell in Eqs. (2)-(6), it is easy to determine the influence of misalignment on the discharge intensity.

\section{SUMMARY OF THE FORMALISM AND NUMERICAL APPLICATIONS}

The ignition of the discharge takes place for magnetic fields greater than $B_{i}$

$$
B_{i}=300 / r_{\mathrm{a}} \text {. }
$$

Transition to HMF-mode takes place at

$$
B_{\text {tr }}=\frac{7.63 \sqrt{ } U_{a}}{r_{\mathrm{a}} P^{0.05}}
$$

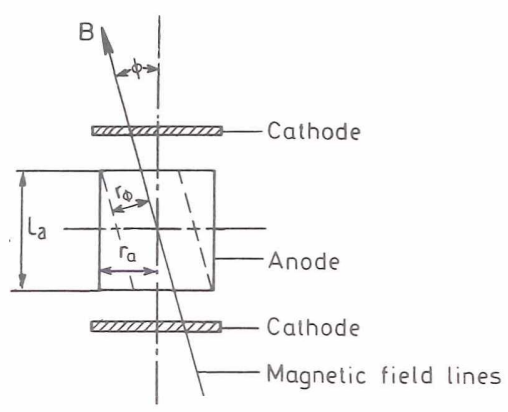

FIG. 4. The decrease of the effective cell radius due to misalignment of cell axis to magnetic field.
Nitrogen pumping speed of one cell:

(i) LMF-mode, $B_{i} \leq B \leq 2 B_{i}$,

$$
S_{1}=6.27 \times 10^{-5}\left(1-\frac{1.5 \times 10^{6} P}{1+4 \times 10^{6} P}\right) P^{0.22 \gamma_{\mathrm{a}}{ }^{2} B_{i}\left(B-B_{i}\right)}
$$

(ii) $\mathrm{LMF}$-mode, $2 B_{i} \leq B \leq B_{\mathrm{tr}}$,

$$
S_{1}=1.56 \times 10^{-5}\left(1-\frac{1.5 \times 10^{6} P}{1+4 \times 10^{6} P}\right) P^{0.2} 2 r_{\mathrm{a}}^{2} B^{2} ;
$$

(iii) HMF-mode, $B \geq B_{\text {tr }}$,

$$
\begin{aligned}
S_{1}=9.1 \times 10^{-4} & \left(1-\frac{1.5 \times 10^{6} P}{1+4 \times 10^{6} P}\right) P^{0.1} l U_{\mathrm{a}} \\
& \times\left\{1-1.5 \times 10^{4}\left[\left(B-B_{\mathrm{tr}}\right) r_{\mathrm{a}} P\right]^{\frac{1}{2}} / U_{\mathrm{a}}\right\} .
\end{aligned}
$$

Symbols and units are as follow:

$B=$ applied magnetic field in $\mathrm{G}$,

$B_{i}=$ magnetic field at the ignition point in $\mathrm{G}$,

$B_{\text {tr }}=$ magnetic field at the transition point in $\mathrm{G}$,

$l=$ effective height of the cell in $\mathrm{cm}$,

$P=$ pressure in Torr,

$r_{\mathrm{a}}=$ cell radius in $\mathrm{cm}$,

$S_{1}=$ nitrogen pumping speed of one cell in $1 / \mathrm{sec}$,

$U_{\mathrm{a}}=$ anode voltage in $\mathrm{V}$.

In an actual sputter-ion pump, the pumping units consist of $n$ cells connected in parallel to the same anode potential (Fig. 5). Thus

$$
S_{n}=n S_{1}
$$

where $S_{n}$ is the pumping speed of the $n$ cells. This pumping speed is lowered to $S_{\text {eff }}$ due to the conductance of the gaps between the anode and the two cathodes. ${ }^{1,13,21}$

$$
S_{\text {eff }}=S_{n} \frac{\tanh D}{D}
$$

with

$$
D=\frac{k a}{7.85 \delta} \sqrt{\frac{S_{n}}{a b}},
$$

where $a$ is the depth of a pumping unit in $\mathrm{cm} ; b$, length of the pumping unit in $\mathrm{cm} ; k$, the factor taking the

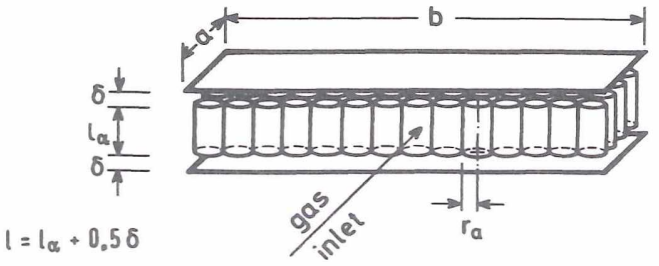

Fig. 5. Electrode structure of a sputter-ion pump element and its characteristic dimensions. 
value 1 when the pumping unit is open to one side and the value $\frac{1}{2}$ for two open sides; and $\delta$, the gap between anode and one cathode in cm (Fig. 5).

If the sputter-ion pump consists of $N$ pumping units, the effective pumping speed on the pump flange $S_{p}$ can be calculated by the equation

$$
\frac{1}{S_{p}}=\frac{1}{N S_{\text {eff }}}+\frac{1}{L}
$$

where $L$ is the conductance of the pump chamber to the connecting flange. The pumping speed $S_{p}$ can be also calculated with more computational effort, using the matrix calculations ${ }^{14}$ proposed by Pisani. ${ }^{15,16}$ Equations (11)-(17) can be also used for carbon monoxide. The proposed formalism is not extended to inert gases and hydrogen due to the complicated pumping speed dependence on the cathode material. ${ }^{17,18}$

The saturated pumping speed is needed in most practical cases for users and manufacturers of sputterion pumps. It is defined as the pumping speed after a quantity of $2 \times 10^{-2} S$ Torr 1 gas has been pumped $(S=$ nominal pumping speed in $1 / \mathrm{sec}) .{ }^{19}$ This saturation for instance can be achieved by operating the pump at $1 \cdot 10^{-6}$ Torr for $5.5 \mathrm{~h}$. To calculate the saturated pumping speed, Eqs. (13)-(15) must be multiplied by the factor $\left(0.75-2 \times 10^{-10} / P\right)$. The first term of this empirical factor expresses a decrease of pumping speed by $25 \%$ due to saturation. The second term is significant for pressures lower than $10^{-8}$ Torr, and it takes into account the decrease of the effective pumping speed due to the outgassing of the pump chamber walls and electrode surfaces. This factor is only valid for pressures greater than $3 \cdot 10^{-10}$ Torr. At this pressure, the effective saturated pumping speed is nearly zero due to thermal outgassing of the gas-covered surfaces.

Using the proposed formalism, the pumping speed of the prototypes of distributed sputter-ion pumps of $\mathrm{SPEAR}^{2}$ was calculated at $5 \times 10^{-7}$ Torr and plotted together with the measured values in Fig. 1. The good

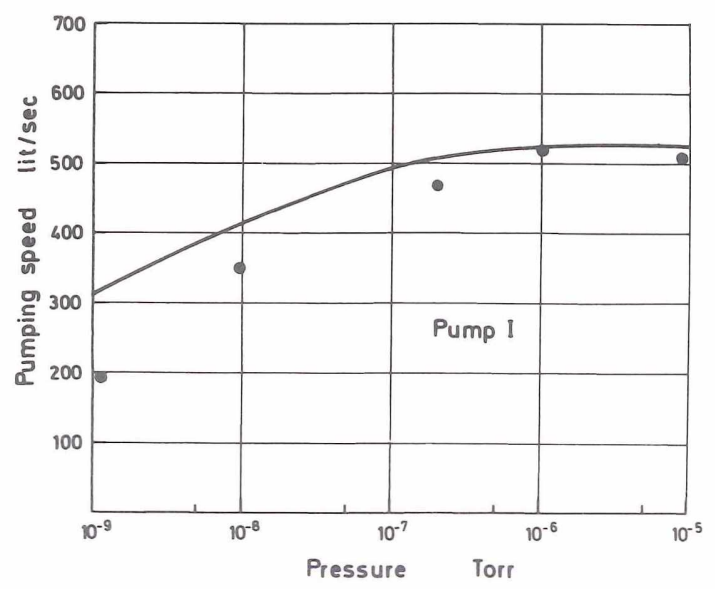

FIG. 6. The pumping speed pressure dependence of the 4001 /sec conventional sputter-ion pumps, installed in the vacuum system of DORIS. - measured not saturated pumping speed; - calculated pumping speed as proposed in this work.

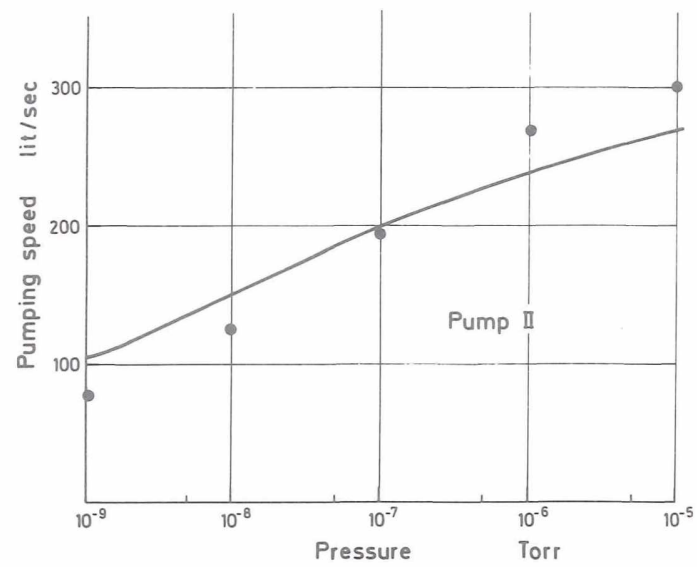

FIG. 7. The pumping speed pressure dependence of a conventional sputter-ion pump (II). - measured not saturated pumping speed; - calculated pumping speed as proposed in this work.

agreement can be observed. The existing deviation of $25 \%$ for the $\phi 1.25 \mathrm{~cm}$ cells at high magnetic fields lies within the accuracy of the pumping speed measurements. In another publication of the SPEAR-group, ${ }^{20}$ the maximum pumping speed of the $\phi 1.25 \mathrm{~cm}$ elements is rated with $500 \mathrm{l} / \mathrm{sec}$. This value is close to our calculations. Two other numerical applications of the formalism are demonstrated in Figs. 6 and 7 for two typical diode pumps with titanium cathodes, rated at $400 \mathrm{l} / \mathrm{sec}$. In pump I, $25 \%$ of the cathode material was tantalum in order to increase the argon pumping speed. Pump I has been installed as standard sputter-ion pump in the vacuum system of the DORIS ${ }^{26}$ storage ring. The values of pump parameter are given in Table I. Both pumps are working in the LMF-mode up to $10^{-6}$ Torr. In this case, the pumping speed depends on the value of magnetic field $\left(\propto B^{2}\right)$. To avoid deviations, due to different magnetic field values at different cells, the mean-square-root of the magnetic field over the whole surface of the element was taken into account. The chamber conductance $L$ was estimated from the dimensions of the pump chamber, using Clausing's equation. ${ }^{23}$ The agreement between calculated and measured values, depending on the accuracy of the pumping speed measurements, is better than $30 \%$. Equation (17) was used for conductance corrections in the pump elements in all numerical applications. The corrections was always less than $25 \%$ of the pumping speed of an element.

TABLE I. Parameters of two tested pumps.

\begin{tabular}{lll}
\hline \hline & \multicolumn{1}{c}{ Pump I } & \multicolumn{1}{c}{ Pump II } \\
\hline$N$ & 4 elements & 16 elements \\
$L$ & $1200 \mathrm{l} / \mathrm{sec}$ & $1200 \mathrm{sec}$ \\
$n$ & $123 \mathrm{cells} /$ element & $8 \mathrm{cells} /$ element \\
$r_{a}$ & $1.03 \mathrm{~cm}$ & $1.25 \mathrm{~cm}$ \\
$l_{a}$ & $2.54 \mathrm{~cm}$ & $2.0 \mathrm{~cm}$ \\
$\delta$ & $1.22 \mathrm{~cm}$ & $0.8 \mathrm{~cm}$ \\
$a$ & $13.3 \mathrm{~cm}$ & $7 \mathrm{~cm}$ \\
$b$ & $43 \mathrm{~cm}$ & $10.6 \mathrm{~cm}$ \\
$B$ & $1090 \mathrm{G}$ & $1000 \mathrm{G}$ \\
$U_{a}$ & $5000 \mathrm{~V}$ & $7500 \mathrm{~V}$ \\
\hline \hline
\end{tabular}




\section{ACKNOWLEDGMENTS}

The authors are indebted to D. Degèle, J. Susta, and K. Steffen for helpful suggestions and to J. Lehmitz and M. G. Meczulat for their skillful technical assistence on pumping speed measurements.

${ }^{1}$ M. D. Malev and E. M. Trakhtenberg, Preprint 280, Inst. Nucl. Phys. Novosibirsk (1969).

${ }^{2}$ U. Cummings et al., J. Vac. Sci. Technol. 8, 348 (1971).

${ }^{3}$ V. V. Ryabov and G. L. Saksagansky, Vacuum 22, 191 (1971).

${ }^{4}$ Vorschlag zum Bau eines $3 \mathrm{GeV}$ Elektron-Positron-Doppelspeicherringes für das Deutsche Elektronen-Synchrotron, (DESY, Hamburg, 1967), p. 55.

${ }^{6}$ M. Bernardini and L. Malter, J. Vac. Sci. Technol. 2, 130 (1965).

${ }^{6} \mathrm{~W}$. Schuurman, Invest. of a Low-Pressure Penning Discharge,

Rijnhuizen Report 66-28 (FOM-Instituut voor Plasma-Fysica, Rijnhuizen, Netherlands, 1966).

${ }^{7}$ W. Bächler, Third Int. Vac. Congr. Stuttgart 2, 609 (1967).

${ }^{8}$ R. L. Jepsen, Le Vide 80, 80 (1959).
9S. L. Rutherford, Trans, 10th AVS Vacuum Symposium (Macmillan, New York, 1963), p. 185

${ }^{10}$ A. Dallos and F. Steinrisser, J. Vac. Sci. Technol. 4, 6 (1967),

${ }^{11}$ A. Dallos, Vacuum 14, 79 (1969).

$12 \mathrm{E}$. Fischer and H. Mommsen, ISR-VAC/66-11 (CERN, 1966).

${ }^{13} \mathrm{G}$. A. Vasil'ev, Pribori i Technika Eksperimenta, 1, 147 (1965).

${ }_{14} \mathrm{G}$. Paul, Vakuum Technik 22, 243 (1973).

${ }^{15}$ C. Pisani, Vacuum 18, 327 (1968).

${ }^{16} \mathrm{C}$. Pisani, Proc. 4th Intern. Vacuum Congr. (Institute of Physics, Manchester, 1968), No. 5, p. 439.

${ }_{17}$ P. N. Baker and L. Laurenson, J. Vac. Sci. Technol. 9, 375 (1972).

${ }_{18}$ J. H. Singleton, J. Vac. Sci. Technol. 6, 316 (1969).

${ }^{19}$ ISO-Proposal, (ISO/TC112/SC3, 1970).

${ }^{20}$ B. Richter, Kerntechnik 12, 531 (1970).

${ }^{21}$ M. D. Malev and E. M. Trakhtenberg, Vacuum 23, 403 (1973).

${ }^{22}$ R. I. Jepsen, Proc. 4th Intern. Vacuum Congr. (Institute of Physics, Manchester, 1968), No. 5, p. 317.

${ }^{23}$ P. Clausing, Ann. Physik (5) 12, 961 (1932).

${ }^{24}$ SPEAR - Stanford Positron-Electron Asymmetric Ring of the Stanford Linear Accelerator Center, Stanford University, Stanford, CA 94304.

${ }^{25}$ DESY - Deutsches Electronen SYnchrotron, Hamburg,

Germany.

${ }^{26}$ DORIS-DOppel Ring Speicher (DESY, Hamburg, Germany). 

